\title{
A Case Series of Transmigrated Canine - Uncovering the Hidden Iceberg
}

\author{
Harshil Naresh Joshi ${ }^{*}$, Deepa Patil2 ${ }^{2}$ Rameshwar H Taluja ${ }^{3}$, Swetha R Taluja ${ }^{4}$, \\ Manjot Kaur ${ }^{5}$ and Shireen Mann ${ }^{6}$ \\ ${ }^{1,2} \mathrm{~K}$ M. Shah Dental College and Hospital, Sumandeep Vidyapeeth, \\ Pipariya, Waghodia, vadodara, Gujarat, India \\ ${ }^{3} B D S, M D S$ (Prosthodontist and Implantologist) \\ ${ }^{4} B D S$, PGCOI( Dental Surgon) \\ ${ }^{5}$ BDS, Baba Jaswant Singh Dental College \& Hospital, Punjab, India \\ ${ }^{6} B D S$,Department of Periodontology and Oral Implantology, Nation Dental College and Hospital, Punjab \\ Corresponding author email: drharshiljoshi11@gmail.com
}

\begin{abstract}
Transmigration of Canines is a rare occurrence. The prevalence of transmigration varies between 0.1 to $0.34 \%$. The teeth commonly involved with transmigration are mandibular canines. Transmigration of maxillary canines is infrequent with very few cases published in the literature. The transmigrated tooth frequently drifts mesially and migrates to the contra lateral side of the arch. Transmigration can be asymptomatic or cause root, malalignment and neural abnormalities. It is usually associated with a retained deciduous tooth or agenesis of adjacent teeth. In this case series we report seven cases of transmigration of canines encountered during orthodontic treatment among which one case involved the maxilla. Panoramic radiograph is an indispensable tool in early diagnosis of transmigration and should be advised in cases of retained deciduous or missing teeth for appropriate patient management Cone beam computed tomography can aid in assessing three-dimensional location, labial and lingual displacement and root resorption in transmigration cases.
\end{abstract}

KEY WORDS: PREVALENCE OF TRANSMIGRATION, MAXILLARY CANINES, CONE BEAM.

\section{INTRODUCTION}

An impacted tooth is defined by Mead as a "tooth which is prevented from erupting into position because of lack of space, malposition, or other impediments' (Archer et al., 1966). According to Farman impacted teeth are prevented from eruption due to a physical barrier within the path of eruption (Agarwal et al., 2004). Maxillary canines are the most commonly impacted teeth (0.8-3.6\%), second only to the third molars (Aydin et al., 2004). The prevalence of mandibular canine impaction is comparatively less common than maxillary canine impactions (Rohrer, 1929). The canines are the longest teeth in the oral cavity. Therefore, the canine teeth should be evaluated thoroughly to deliver the best treatment to the patients (Jain and Debbarma.

Biosc Biotech Res Comm P-ISSN: 0974-6455 E-ISSN: 2321-4007

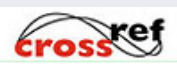

Identifiers and Pagination

Year: 2021 Vol: 14 No (6) Special Issue

Pages: 14-29

This is an open access article under Creative

Commons License Attribn 4.0 Intl (CC-BY). DOI: $h t t p: / / d x$.doi.org/10.21786/bbrc/14.6.3
Sporadically, an unerupted tooth drifts to a newer location from the site of origin but on the ipsilateral side of the arch. Infrequently, an impacted canine migrates to the contralateral side of the arch, crossing the midline. This anomalous movement of a tooth has been termed transmigration. Formerly, the phrase transmigration was employed when the whole impacted canine had drifted and crossed the midline of the mandible (Tarsitano et al., 1971). Later Javid emphasized 'transmigration as a condition where one half or more of impacted canine crosses the midline'(Javid, 1985). According to various studies, the prevalence of transmigration is suggested to be 0.1 to $0.34 \%$ in different populations (Jain andDebbarma, 2019; Kamiloglu et al., 2014; Aktan et al., 2010; Gündüz et al., 2010).

A retrospective analysis by M Mupparapu et al (2002) 11investigated the patterns of transmigration and eruption of permanent mandibular canines \&t mechanisms affecting the eruption path. They classified the patterns of mandibular canine transmigration into five types. The occurrence of transmigration is a rarity and very few case series have been reported among the Indian population. We are hereby reporting seven cases of

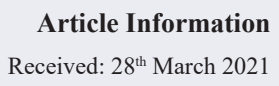
ccepted after revision: $26^{\text {th }}$ May 2021 
Canine transmigration amongst patients reporting for orthodontic treatment.

\section{Case reports}

Case - 1: A 21-year-old female patient, reported to the department of Orthodontics complaining of irregularly placed upper \& lower front teeth. On intraoral examination, the left mandibular deciduous canine was over-retained. Panoramic radiograph (Figure 1) revealed transmigration of left mandibular permanent canine crossing the midline and located below the apices of the right mandibular canine at the lower border of the mandible. The tip of the mandibular canine was facing downwards and impinging the lower cortical border of the mandible. There were no associated symptoms of paraesthesia or pain. There was no evidence of root resorption with $73 \& 43$. The right maxillary canine was also impacted. (13) This was classified as Type 2 Canine transmigration.
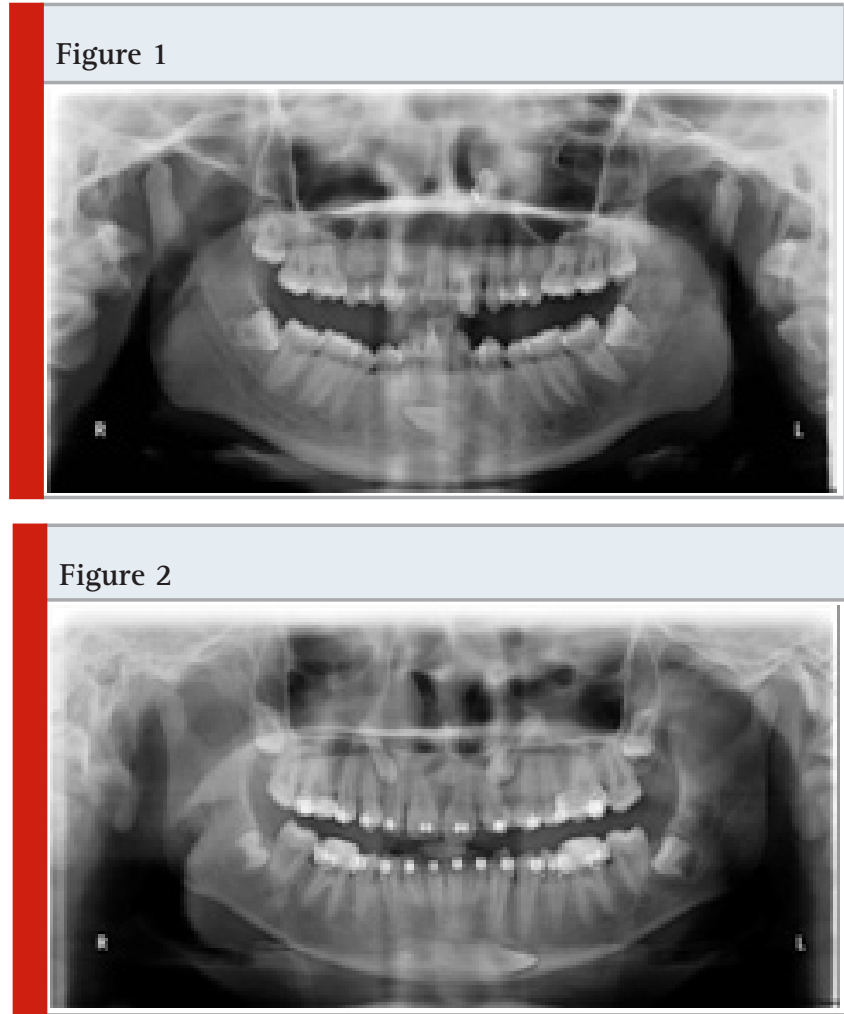

Case - 2: Many hypotheses have been suggested for transmigration and include trauma, long-distance for eruption, and pathological lesions (Kamiloglu et al., 2014; Aktan et al., 2010). Transmigration can cause pressure resorption of adjacent roots resulting in neurologic symptoms. The adjacent teeth can be drifted and sometimes displaced to the coronoid process causing pain and discomfort to the patient (Gündüz et al., 2010). Majority of the times, the transmigrated tooth remains impacted \& is asymptomatic. A 19-year-old female patient reported to the department of orthodontics with a chief complaint of forwardly placed upper front teeth. On intraoral examination, there was the presence of over-retained 83. The panoramic radiograph revealed the presence of right mandibular canine (43) apical to the mesial aspect of tooth left mandibular canine(33) (Figure 2). The migrating canine 43 showed no evidence of resorption or pericoronal radiographic changes suggestive of cystic degeneration. Adjacent teeth appeared normal. This was classified as Type 1 canine transmigration.

Case - 3: A 22-year-old female patient reported to the orthodontics department complaining of missing teeth in the lower right anterior region of the jaw. The clinical history did not reveal any past dental history and there was no previous history of trauma. On clinical examination, the left mandibular canine was missing. As a part of the routine assessment, a panoramic radiograph was taken, which revealed the presence of an impacted transmigrated canine in a horizontal position below the root apices of lower incisors impinging the lower border of the mandible. This was classified as Type 2 Canine transmigration.

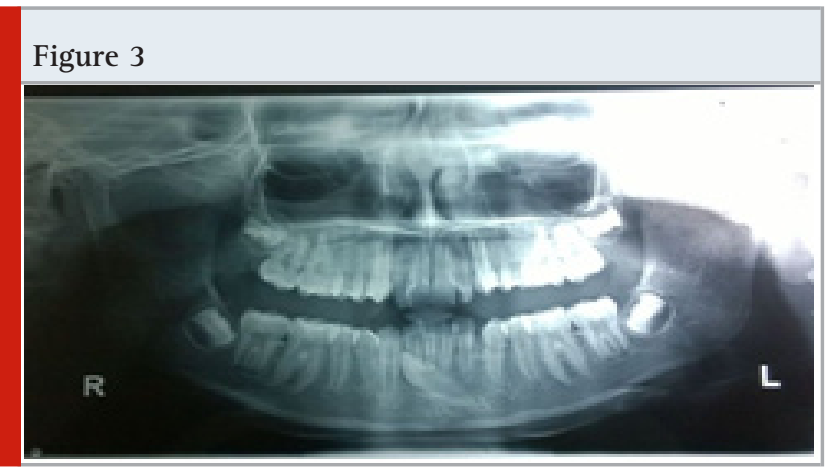

Case - 4: A 14-year-old male patient was referred for an orthodontic opinion regarding a missing lower canine tooth. On Intraoral examination, he had an intact dentition, apart from a missing left mandibular canine (33). The panoramic radiograph showed mesially drifted 33 to the contralateral side. The tip of the canine crown is visible between 43 \& 44 . (Figure 4) Due to the superimposition of 33 on the roots of 43, the root resorption was not evident. This was classified as Type 1 canine transmigration.

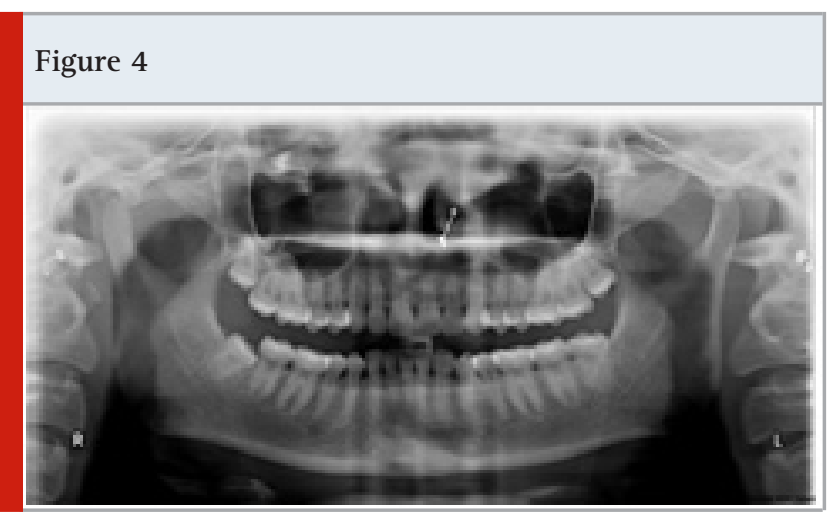

Case - 5: A15-year-old male patient reported to the orthodontics department for ongoing Orthodontic treatment. On intraoral examination 13,23,43 was missing. The panoramic radiograph revealed impacted right and left maxillary canine (13 \&t 23). The right 
mandibular canine (43) transmigrated from the right to the left side of the mandible. This was classified as Type 2 Canine transmigration.

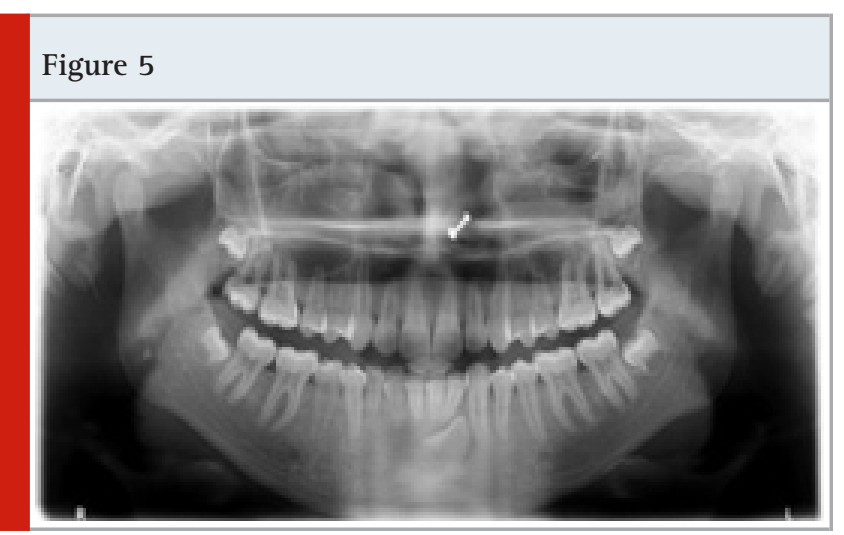

Case - 6: An 18-year-old female patient reported to the orthodontics department with malaligned lower anterior teeth. The clinical history did not reveal any past dental history and there was no previous history of trauma. On clinical examination, severe crowding was seen $\&$ the left mandibular canine was missing. The panoramic radiograph revealed the presence of an impacted transmigrated left mandibular canine (33) placed obliquely below the root apices of lower incisors. This was classified as Type 2 Canine transmigration.

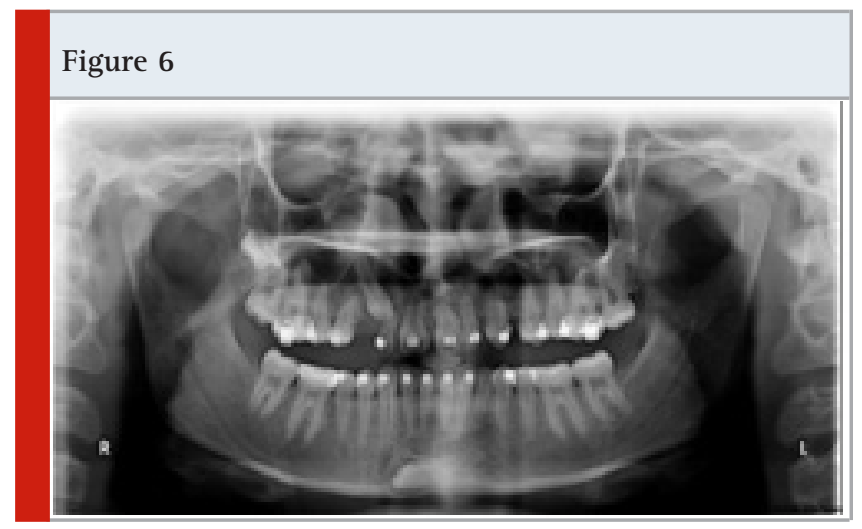

Figure 7

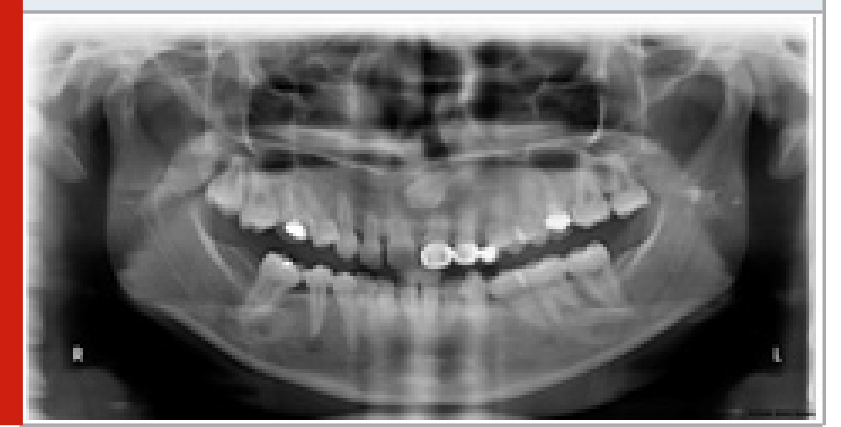

Case-7: A 25-year old male patient reported for evaluation of his missing teeth. The panoramic radiograph revealed transmigrated left maxillary canine between the roots of maxillary central incisors. There was an increase in the follicular size surrounding the left maxillary canine and there was no clear evidence of root resorption of maxillary central incisors. Table 1 explains the salient features observed in the transmigrated canine cases. No active treatment was performed as the patients were asymptomatic. The patients were advised periodic follow-up and panoramic radiograph every six months to monitor the changes around the transmigrated canine.

\section{DISCUSSION}

Ando et al. were the early pioneers to study the process of transmigration of mandibular canines. They took several sequential radiographs at regular intervals for many years and observed the lower canines traversing the mandibular symphysis to the other side of the dental arch. They were also the first to label the term 'transmigration'.12 The most common teeth associated with transmigration are the permanent canines. The mandibular canines are commonly associated with transmigration as compared with the maxillary canines. Infrequently, mandibular premolars and lateral incisors are transmigrated (.079\%) (Kamiloglu et al., 2014) The prevalence of transmigration in the Indian population ranges from $0.1-0.5 \%$ (Jain and Debbarma, 2019; Sharma et al., 2014). In the present series, 7 cases of transmigration were recorded after examining the panoramic radiograph of orthodontic patients reported for one year.

Transmigration is commonly reported as an incidental finding on panoramic radiographs or during clinical evaluation of missing teeth, between the age group of 8 to 69 years. 5 In the present case series, the patient's age ranged between 14-25 years, which is the most common age group for orthodontic treatment. The present case series showed a slight female predominance (4FM >3M) which has been substantiated in previous studies by Aydin et al. (2004) and Celikoglu et al. (2010).

However, in a study by Aras et al15, there was no gender predilection in his 12 reported transmigration cases. The increased prevalence of canine transmigration in the mandible could be due to the larger cross-sectional area available in the anterior mandible in contrast to the anterior maxilla. Transmigration of maxillary canines is a rarity owing to the proximity between the roots of maxillary incisors and the floor of the nasal fossa which acts as a barricade for migration. Additional impediments for transmigration in the maxilla include the presence of midpalatal suture and roots of adjacent teeth (Aydin et al., 2004). In the present case series, a single case of maxillary canine transmigration was reported which is a rare phenomenon. The left maxillary canine is impacted and has migrated between the roots of the maxillary central incisors. Very few cases of maxillary canine transmigration have been reported in the literature by Jain et al. (2019), Celikoglu et al. (2010) and Aras et al. (2008).

Various theories have been proposed for transmigration and have been mentioned below.

1. The presence of canine tooth bud in front of mandibular incisors coupled with facial growth 
thrusts the canine in the opposite direction of the mandibular arch (Auluck et al., 2006).

2. Few other investigators believed that a robust eruption force or a deflection of the crypt of the tooth bud might lead to an ectopic eruption. 14

3. Abnormal displacement of dental lamina during embryonic life (Tarsariya et al., 2015)..

4. According to Vichi and Franchi (1991), congenitally missing teeth like lateral incisor or over retention of the primary canine cause an improper path for an eruption of permanent canine. The canine then migrates from its original site to a horizontal or oblique position through the symphyseal bone in front of the mandibular incisors depending on the available space.

5. Occasionally, certain pathologies have been associated with transmigration including, fracture, cysts, odontomas, and Gardner's syndrome. The transmigrated canines may also develop a cystic lesion similar to impacted teeth (Taguchi et al., 2001; Auluck et al., 2006; Celikoglu et al., 2010).
6. A regional disturbance in the dental follicle may lead to local defective osteoclastic function with an abnormal eruption pathway being formed. This is a probable explanation for an aberrant eruption of teeth (Auluck et al., 2006).

In the present case series, two cases were associated with over-retained primary canines thus justifying the occurrence of transmigration. Impacted teeth may migrate mesially or distally in the bone. The movement of impacted teeth is along the route of the crown and this could be the reason for the transmigration of canines to the contralateral side. 9Transmigrated teeth preserve their nerve connection to the originating site where the tooth germ is formed. Therefore, it is imperative to anesthetize the nerve from the region of origin (Sharma and Nagpal, 2011). A single case report discussed an incidence of pain experienced by a patient during surgical removal of a transmigrated canine as the inferior alveolar nerve of the opposite side (original site of the transmigrated canine) was not anesthetized (Gunashekhar and Rohini, 2011).

Table 1. Tabular representation of the transmigrated canine cases

\begin{tabular}{|l|c|l|l|l|l|c|}
\hline S.No. & Age (years) & Gender & Location & Right/Left & $\begin{array}{c}\text { Type of } \\
\text { transmigration }\end{array}$ & $\begin{array}{c}\text { Associated } \\
\text { anomalies }\end{array}$ \\
\hline 1 & 21 & Female & Mandible & Left & Type 2 & $\begin{array}{c}\text { Over retained 73, } \\
\text { Impacted 13 }\end{array}$ \\
\hline 2 & 19 & Female & Mandible & Right & Type 1 & Over retained 83 \\
\hline 3 & 22 & Female & Mandible & Left & Type 2 & - \\
\hline 4 & 14 & Male & Mandible & Left & Type 1 & - \\
\hline 6 & 15 & Male & Mandible & Right & Type 2 & Impacted 13ct 23 \\
\hline 7 & 18 & Female & Mandible & Left & Type 2 & - \\
\hline
\end{tabular}

Most of the cases of transmigration are unilateral as seen in the present case series and substantiated in the literature (Sharma and Nagpal, 2011; Auluck et al., 2006; Celikoglu et al., 2010; Gündüz and Çelenk, 2010). In our study left transmigrated canines were found more frequently than right canines. In a study by Aras et al. (2008) the, transmigrated canines were similar in number on both sides.Celikoglu14 et al in their study observed a predominance on the left side. The dissimilarity could be due to the analysis of the general population in other studies and the orthodontic population in our study. Very few cases of bilateral transmigration have been reported in the literature. The bilateral existence of transmigrated canine was first described by Joshi et al22. Mupparapu had used five criteria to classify the transmigrated canines Type 1- 5 (Ando et al., 1964).

Type 1 . The canine is impacted mesioangular across the midline, labial, or lingual to the anterior teeth with the crown portion of the tooth crossing the midline.

Type 2. The canine is horizontally impacted near the inferior border of the mandible below the apices of incisors.

Type 3. The canine has erupted either mesial or distal to the opposite canine.

Type 4. The canine is horizontally impacted near the inferior border of the mandible below the apices of either premolars or molars on the opposite side.

Type 5 . The canine is positioned vertically in the midline with the long axis of the tooth crossing the midline.

Most of the cases reported in the literature are Type 1 (Aydin et al., 2004; Mupparapu, 2002; Celikoglu et al., 2010; Auluck et al., 2006; Sharma and Nagpal, 2011) ,. In the present case series, 3 were of Type 1 and 3 of Type 2 and a single case of maxillary canine transmigration was observed. Most of the cases of transmigration are asymptomatic. Few cases of sensory alteration and infection have been reported when the canines erupt in the midline and cause root resorption of adjacent teeth. The transmigrated canines can cause root resorption of adjacent teeth. In the present case series, all the patients 
were asymptomatic and there was no evidence of root resorption of adjacent teeth on a panoramic radiograph (Auluck et al., 2006; Celikoglu et al., 2010; Aras et al., 2008).

The low prevalence of root resorption reported in the literature could be due to the distortion seen in the mandibular anterior region on the panoramic radiograph. This can be overcome by prescribing Cone-beam computed tomography (CBCT) in transmigration cases. CBCT can accurately identify the location of canines, their labial and lingual position, and the presence of root resorption (Bertl et al., 2018).

The type of transmigrated canine radiographically and the presence of clinical symptoms are detrimental in deciding their treatment. The various treatment options include surgical extraction, autotransplantation, and surgical exposure combined with orthodontic alignment.A surgical extraction is preferred in cases of root resorption, infections, cystic transformation, and presence of nerve impingement. Transplantation of the canine is indicated when the mandibular incisors are properly aligned with sufficient space to move the canine to its original location (Sharma and Nagpal, 2011; Aras et al., 2008). In the present case series, no treatment was performed as the patients were asymptomatic and there was no evidence of root resorption. Howard (1976) 'transplanted a transmigrant canine into its normal position in the arch since the required amount of space had been preserved'.

Orthodontic treatment has been tried to bring the transmigrated canines but it is difficult to get the transmigrated canines back to their original positions after they have transmigrated to the contralateral side (Wertz, 1994; Camilleri and Scerri, 2003). Follow up and radiographic monitoring is recommended among asymptomatic patients, proximity to vital structures, and patients not willing for treatment. In the present case series, no treatment was instituted and the patients were on regular follow up for clinical and radiographic monitoring.

\section{CONCLUSION}

The present case series demonstrated a rare phenomenon of transmigrated canines among orthodontic patients. Over retention of deciduous canines and congenitally missing teeth should be evaluated and screened with panoramic radiograph for early detection and management of transmigrated canines. The transmigrant canines can be asymptomatic or cause root resorption of adjacent teeth with neurological impairment. Treatment depends on the position of transmigrated canines and the presence of symptoms. Advanced imaging modalities like $\mathrm{CBCT}$, can provide precise data regarding their location and proximity to vital structures. Future studies should be carried out to assess the early incidence of transmigrated canines and longitudinal studies should be undertaken to assess the outcome of early orthodontic treatment on transmigrated canines.

\section{REFERENCES}

Agarwal, K.N., Gupta, R., Faridi, M.M. and Kalra, N., 2004. Permanent dentition in Delhi boys of age 5-14 years. Indian Pediatr, 41(10), pp.1031-5.

Ajit Auluck, M.D.S., Archna Nagpal, M.D.S., Suhas Setty, M.D.S., Keerthilatha, M.P. and Sunny, J., 2006. Transmigration of impacted mandibular canines-report of 4 cases. J Can Dent Assoc, 72(3), pp.249-52.

Aktan, A.M., Kara, S., Akgünlü, F. and Malkoç, S., 2010. The incidence of canine transmigration and tooth impaction in a Turkish subpopulation. The European Journal of Orthodontics, 32(5), pp.575-581.

Ando, S., Aizawa, K., Nakashima, T., Sanka, Y., Shimbo, K. and Kiyokawa, K., 1964. Transmigration process of the impacted mandibular cuspid. The Journal of Nihon University School of Dentistry, 6(2), pp.66-71.

Aras, M.H., Büyükkurt, M.C., Yolcu, Ü., Erta, Ü. and Dayi, E., 2008. Transmigrant maxillary canines. Oral Surgery, Oral Medicine, Oral Pathology, Oral Radiology, and Endodontology, 105(3), pp.e48-e52.

Archer, W.H., 1966. Impacted Teeth. Oral Surgery: A Step-by-Step Atlas of Operative Techniques.

Aydin, U., Yilmaz, H.H. and Yildirim, D., 2004. Incidence of canine impaction and transmigration in a patient population. Dentomaxillofacial Radiology, 33(3), pp.164-169.

Bertl, M.H., Frey, C., Bertl, K., Giannis, K., Gahleitner, A. and Strbac, G.D., 2018. Impacted and transmigrated mandibular canines: an analysis of 3D radiographic imaging data. Clinical oral investigations, 22(6), pp.2389-2399.

Camilleri, S. and Scerri, E., 2003. Transmigration of mandibular canines-a review of the literature and a report of five cases. The Angle Orthodontist, 73(6), pp.753-762.

Celikoglu, M., Kamak, H. and Oktay, H., 2010. Investigation of transmigrated and impacted maxillary and mandibular canine teeth in an orthodontic patient population. Journal of Oral and Maxillofacial Surgery, 68(5), pp.1001-1006.

Gunashekhar, M. and Rohini, M., 2011. Transmigration of mandibular canines: a rare case report and review of literature. Journal of Dentistry for Children, 78(1), pp.19-23.

Gündüz, K. and Celenk, P., 2010. The incidence of impacted transmigrant canines: a retrospective study. Oral Radiology, 26(2), pp.77-81.

Howard, R.D., 1976. The anomalous mandibular canine. British Journal of Orthodontics, 3(2), pp.117-121.

Jain, S. and Debbarma, S., 2019. Patterns and prevalence of canine anomalies in orthodontic patients. Medicine and pharmacy reports, 92(1), p.72.

Javid, B.R., 1985. Transmigration of impacted mandibular cuspids. International journal of oral surgery, 14(6), pp.547-549.

Joshi, M.R. and Shetye, S.B., 1994. Transmigration of mandibular canines: a review of the literature and report 
of two cases. Quintessence international, 25(4).

Kamiloglu, B. and Kelahmet, U., 2014. Prevalence of impacted and transmigrated canine teeth in a Cypriote orthodontic population in the Northern Cyprus area. BMC Research notes, 7(1), pp.1-6.

Latchoumi, T.P., Ezhilarasi, T.P. and Balamurugan, K., 2019. Bio-inspired weighed quantum particle swarm optimization and smooth support vector machine ensembles for identification of abnormalities in medical data. SN Applied Sciences, 1(10), pp.1-10.

Mupparapu, M., 2002. Patterns of intra-osseous transmigration and ectopic eruption of mandibular canines: review of literature and report of nine additional cases. Dentomaxillofacial Radiology, 31(6), pp.355-360.

Röhrer, A., 1929. Displaced and impacted canines A radiographic research. International Journal of Orthodontia, Oral Surgery and Radiography, 15(10), pp.1003-1020.

Sharma, G. and Nagpal, A., 2011. Transmigration of mandibular canine: report of four cases and review of literature. Case reports in dentistry, 2011.

Sharma, G. and Nagpal, A., 2014. A study of transmigrated canine in an Indian population. International scholarly research notices, 2014.

Taguchi, Y., Kurol, J., Kobayashi, H. and Noda, T., 2001. Eruption disturbances of mandibular permanent canines in Japanese children. International Journal of Paediatric Dentistry, 11(2), pp.98-102.

Tarsariya, V.M., Jayam, C., Parmar, Y.S. and Bandlapalli, A., 2015. Unusual intrabony transmigration of mandibular canine: case series (report of 4 cases). Case Reports, 2015, p.bcr2014205398.

Tarsitano, J.J., Wooten, J.W. and Burditt, J.T., 1971. Transmigration of nonerupted mandibular canines: report of cases. The Journal of the American Dental Association, 82(6), pp.1395-1397.

Vichi, M. and Franchi, L., 1991. The transmigration of the permanent lower canine. Minerva Stomatologica, 40(9), pp.579-589.

Wertz, R.A., 1994. Treatment of transmigrated mandibular canines. Am J OrthodDentofacOrthop,106:419. 